\title{
PREVENÇÃO DE FALHAS EM SERVIÇOS BANCÁRIOS
}

PREVENTION OF FAILURES IN BANKING SERVICES

Recebimento: 9/02/2019 - Aceite: 30/06/2019 - Publicação: 31/07/2019

Processo de Avaliação: Double Blind Review

\author{
Matheus Gravito Santos ${ }^{1}$ \\ Mestre em Administração pelo Centro Universitário UNA \\ Professor do Centro Universitário UNA \\ ms.gravito@gmail.com
}

\author{
Luiz Rodrigo Cunha Moura \\ Doutor em Administração pela Universidade Federal de Minas Gerais (UFMG) \\ Professor do Centro Universitário UniBH \\ luizrcmoura@gmail.com
}

Cristiana Trindade Ituassu

Doutora em Administração pela Fundação Getúlio Vargas (FGV/SP)

Professora da Universidade Federal de Minas Gerais (UFMG)

cristianaituassu@yahoo.com.br

\section{RESUMO}

O setor bancário está em constante crescimento, assim como a concorrência e as exigências dos clientes que estão aumentando continuamente neste setor. Mesmo para os bancos que prestam serviços de excelência, as falhas durante a prestação de serviços podem ocorrer. Como consequência, quando os serviços falham, os bancos sofrem com o aumento do risco de perderem os seus clientes. Esta pesquisa tem como objetivo por objetivo propor ações de prevenção em serviços bancários com base nas falhas na qualidade do serviço e nas estratégias para o fechamento das lacunas. Para isso, optou-se por um estudo de abordagem qualitativa e quantitativa, com finalidade descritiva em corte transversal, por meio de survey. Além disso, foi desenvolvido um questionário semiestruturado aplicado em clientes que tiveram alguma experiência negativa com bancos nos últimos 12 meses. Os respondentes foram contatos por meio do Facebook, Instagram, Linkedin, Whatsapp ou e-mail. Após a análise dos dados faltantes, a amostra final ficou com 579 questionários válidos. Os dados foram analisados por meio de análise de conteúdo e estatística descritiva. Com os resultados alcançados, foi possível classificar os principais problemas relatados pelos clientes que

\footnotetext{
${ }^{1}$ Autor para correspondência: Centro Universitário UNA: Rua Guajajaras, 175, Centro, Belo Horizonte - MG, Brasil.
} 
vivenciaram experiências negativas com serviços bancários, identificando a gravidade da falha percebida por estes clientes para os problemas relatados. Por fim, foram descritas estratégias para o fechamento dessas lacunas de falha na qualidade dos serviços bancários.

Palavras-chave: Bancos; Serviços; Qualidade; Modelo de Lacunas; Recuperação de Serviços.

\section{ABSTRACT}

The banking sector is constantly growing, as is the competition and customer requirements that are steadily increasing in this industry. Even for banks that provide services of excellence, failures during the provision of services may occur. As a consequence, when services fail, banks suffer from an increased risk of losing their customers. The objective of this research is to propose actions to prevent bank failures based on service quality gap gaps and strategies to close the gaps. For this, a qualitative and quantitative study was chosen, with descriptive purpose in cross-section, through a survey. In addition, a semi-structured questionnaire was developed for clients who had some negative experience with banks in the last 12 months. The respondents were contacts through Facebook, Instagram, Linkedin, Whatsapp or email. After the analysis of the missing data, the final sample had 579 valid questionnaires. Data were analyzed through content analysis and simple descriptive statistics. With the results achieved, it was possible to classify the main problems reported by clients who experienced negative experiences with banking services, identifying the severity of the perceived failure of these clients to the reported problems. Finally, strategies for closing these gaps in the quality of banking services were described.

Keywords: Banks; Services; Quality; Gap Model; Service Recovery.

\section{INTRODUÇÃO}

A concorrência no setor bancário está em constante crescimento junto com as exigências dos clientes desse setor. O objetivo final de um prestador de serviços deve ser exceder as expectativas dos clientes, para aumentar o nível de satisfação destes clientes (REIMANN; LÜNEMANN; CHASE, 2008).

Os clientes mantêm expectativas muito diferentes sobre o nível de serviço prestado, em função da reputação de uma organização (BATESON; HOFFMAN, 2016; ZEITHAML; BITNER; GREMLER, 2014). A reputação sólida de uma organização tem influenciado o tipo resposta dos clientes, incluindo a escolha de produtos e serviços e intenções de compra (HESS JR, 2008). As empresas que possuem altos índices de satisfação dos clientes aumentam a capacidade de se diferenciarem da concorrência (BATESON; HOFFMAN, 2016; MOURA et al., 2019). 


\section{REVISTA ENIAC PESQUISA}

Os clientes percebem a qualidade do serviço avaliando se existe alguma diferença entre aquilo que eles esperam receber - as expectativas - e aquilo que eles percebem que receberam - as percepções (CHEN; HU, 2013; GREEN, 2014; ZEITHAML; BITNER; GREMLER, 2014). A lacuna do cliente é a diferença entre as expectativas e as percepções do cliente e as expectativas do cliente são os padrões ou pontos de referência que ele traz consigo para a experiência do serviço, enquanto as percepções são avaliações subjetivas das experiências reais com o serviço (GREEN, 2014; ZEITHAML; BITNER; GREMLER, 2014).

O modelo de lacunas fornece uma estrutura integrada para gerenciar a qualidade do serviço e a inovação no serviço orientada para o cliente. Além do mais, o modelo de lacunas de Parasuraman, Zeithaml e Berry (1985) desenha uma comparação entre a qualidade de um serviço que um cliente espera receber e o nível real de desempenho do serviço percebido (GREEN, 2014; KONI; ZAINAL; IBRAHIM, 2013).

Considerando as lacunas de falha na qualidade do serviço e nas estratégias para o fechamento das lacunas, existe o interesse em identificar quais ações de prevenção de falhas em serviços podem ser adotadas no setor bancário.

Assim, esse trabalho tem por objetivo propor ações de prevenção de falhas em serviços bancários com base nas lacunas de falha na qualidade do serviço e nas estratégias para o fechamento das lacunas. Busca-se também classificar os principais problemas relatados pelos clientes que vivenciaram experiências negativas com serviços bancários, identificando a gravidade da falha percebida pelos clientes para os principais problemas relatados, correlacionando os problemas relatados pelos clientes com as lacunas de falha na qualidade dos serviços bancários e descrevendo estratégias para o fechamento das lacunas de falha na qualidade dos serviços bancários.

As contribuições teóricas desse trabalho são descritas em termos do maior conhecimento e compreensão sobre a satisfação do cliente, assim como a qualidade do serviço, como sendo uma atitude formada por uma avaliação global do desempenho no longo prazo (BATESON; HOFFMAN, 2016).

A satisfação do cliente é uma consequência da qualidade do serviço e determina o sucesso a longo prazo de uma organização (LIMA; MOURA; SOUKI, 2015; PARASURAMAN; ZEITHAML; BERRY, 1994; REIMANN; LÜNEMANN; CHASE, 


\section{REVISTA ENIAC PESQUISA}

2008). Além disso, essa pesquisa amplia o conhecimento sobre os serviços bancários, haja vista que o setor está passando por grandes transformações.

Em termos práticos, esse trabalho se justifica, pois a experiência satisfatória do cliente é considerada um dos fatores impactantes para o compromisso com a marca e o boca a boca positivo. Uma experiência satisfatória significa aumentar a sustentabilidade e a vantagem competitiva do negócio (KURTULMUŞOĞLU; PAKDIL, 2016; MOURA et al., 2010).

A partir dos resultados desta pesquisa, os gestores terão a visão das principais experiências negativas vivenciadas pelos clientes com serviços bancários, incluindo a gravidade da falha percebida pelos clientes de bancos para os problemas relatados. Além disso, os problemas relatados pelos clientes foram correlacionados com as lacunas de falha na qualidade dos serviços bancários e sugeridas estratégias para o fechamento dessas lacunas, com os gestores atuando de forma proativa com o foco na qualidade dos serviços prestados.

\section{REFERENCIAL TEÓRICO}

\subsection{LACUNAS DE FALHA NA QUALIDADE DO SERVIÇO}

O modelo de lacunas de Parasuraman, Zeithaml e Berry (1985) sugere que quatro lacunas precisam ser preenchidas para que se feche a lacuna das expectativas e percepções do cliente. O objetivo final é fechar essa lacuna ao atender ou exceder as expectativas dos clientes.

Essas quatro lacunas (Figura 1) ocorrem no interior das empresas prestadoras de serviço (chamadas lacunas da empresa) e incluem: Lacuna 1: a lacuna da compreensão do cliente; Lacuna 2: a lacuna do projeto e dos padrões de serviço; Lacuna 3: a lacuna do desempenho do serviço; Lacuna 4: a lacuna da comunicação (GREEN, 2014; PARASURAMAN; ZEITHAML; BERRY, 1985; ZEITHAML; BERRY; PARASURAMAN, 1993; ZEITHAML; BITNER; GREMLER, 2014). 
Figura 1: O modelo de lacunas da qualidade do serviço

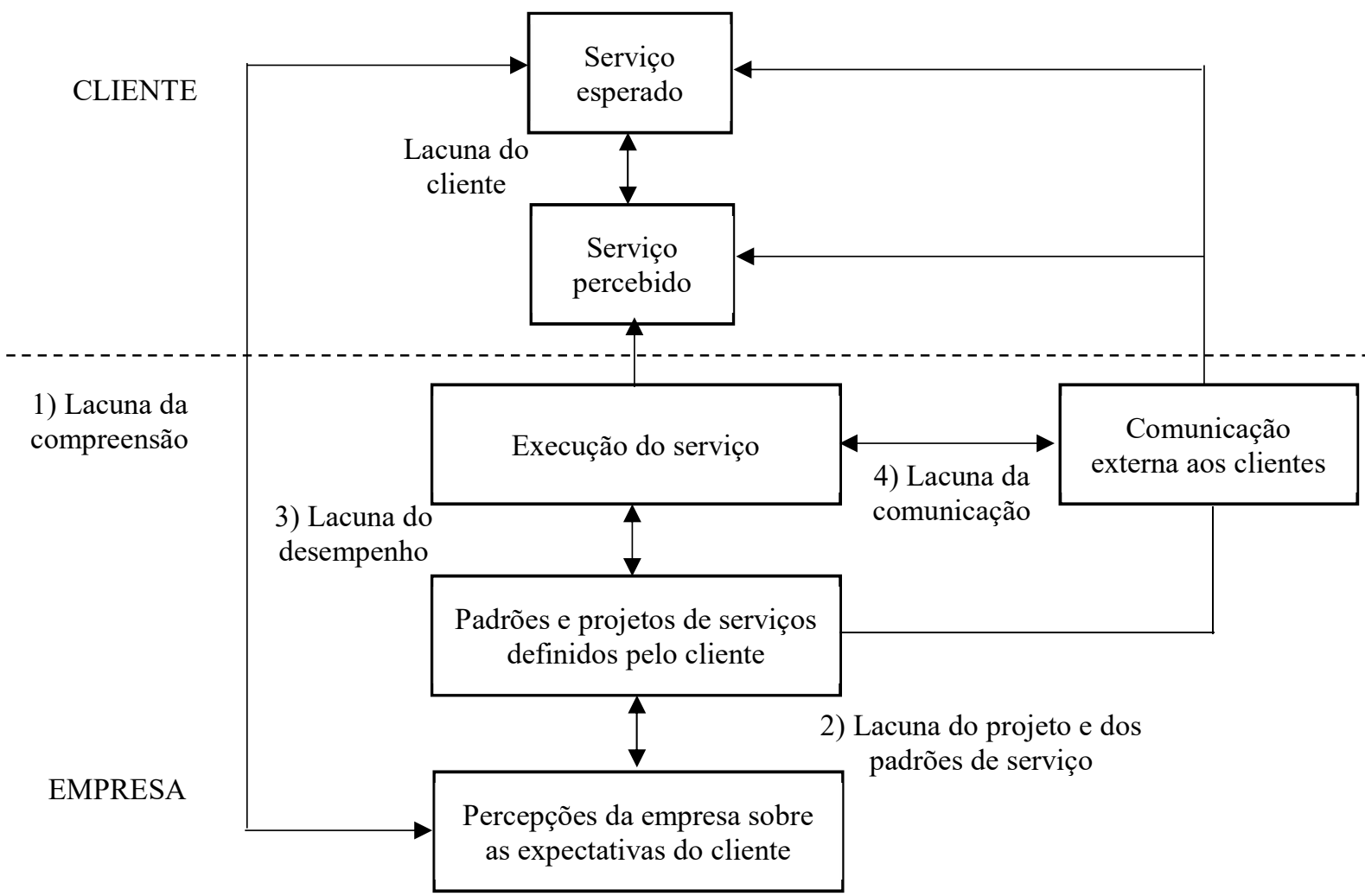

Fonte: ZEITHAML; BITNER; GREMLER, 2014, p. 45 (adaptado pelos autores).

\subsubsection{Lacuna 1: a lacuna da compreensão do cliente}

É a diferença entre as expectativas do cliente quanto ao serviço e a compreensão dessas expectativas por parte da empresa. Uma das principais causas das empresas não atenderem às expectativas dos clientes é a falta de uma compreensão precisa de quais são exatamente essas expectativas (GREEN, 2014; ZEITHAML; BITNER; GREMLER, 2014).

Existem muitos motivos para que os gerentes não estejam conscientes do que os clientes esperam: os gerentes podem não interagir diretamente com os clientes, eles podem não estar interessados em identificar essas expectativas ou podem estar despreparados para abordá-los. O fechamento da lacuna de compreensão exige informações precisas sobre as expectativas dos clientes, as quais devem ser avaliadas com precisão antes de novos serviços serem desenvolvidos e devem ser acompanhadas após a introdução dos serviços (KONI; ZAINAL; IBRAHIM, 2013; ZEITHAML; BITNER; GREMLER, 2014). 


\subsubsection{Estratégias para o fechamento da Lacuna 1 - Compreensão do cliente:}

I. Ouvir os clientes de múltiplas formas, através da pesquisa com o cliente e da comunicação ascendente dos funcionários (TAN; HAMID; CHEW, 2016; ZEITHAML; BITNER; GREMLER, 2014).

II. Criar relacionamentos através da compreensão e atendimento às necessidades dos clientes ao longo do tempo (TAN; HAMID; CHEW, 2016). É uma atividade interpessoal, realizada através de pessoas de contato na linha de frente da empresa de serviços (MOURA, et al., 2019; ZEITHAML; BITNER; GREMLER, 2014).

III. Saber e agir sobre o que os clientes esperam quando experimentam uma falha no serviço, ou seja, aplicar a recuperação do serviço com entendimento sobre essa falha cometida e atuar sobre ela. (TAN; HAMID; CHEW, 2016; ZEITHAML; BITNER; GREMLER, 2014).

\subsubsection{Lacuna 2: a lacuna do projeto e dos padrões de serviço}

Esta lacuna se concentra em traduzir expectativas em projetos de serviço reais, bem como desenvolver padrões para medir as operações de serviços em relação às expectativas dos clientes. As suas principais causas são a ausência de padrões definidos pelo cliente, projeto de serviço deficiente e evidência física e cenário de serviços inadequados (GREEN, 2014; ZEITHAML; BITNER; GREMLER, 2014).

\subsubsection{Estratégias para o fechamento da Lacuna 2 - Projeto e padrões de serviço:}

I. Empregar práticas bem definidas de desenvolvimento de novos serviços e inovação para a concepção de serviços. Um processo formalizado envolve uma série de etapas que começam com a formulação da estratégia (a geração de ideias) e termina com a implementação em larga escala (TAN; HAMID; CHEW, 2016; ZEITHAML; BITNER; GREMLER, 2014).

II. Compreender sobre a experiência total do cliente e desenhar todos os elementos dessa experiência, de forma a atender ou exceder as expectativas do cliente (KONI; ZAINAL; IBRAHIM, 2013; TAN; HAMID; CHEW, 2016). Isso envolve considerar tudo o que ocorre com o cliente, desde o momento em que ele se envolve com o 


\section{REVISTA ENIAC PESQUISA}

serviço e durante todo o período de experiência do serviço (ZEITHAML; BITNER; GREMLER, 2014).

Os elementos comuns da experiência de serviço que precisam ser projetados, incluem processos voltados para o cliente, o espaço físico onde o serviço é entregue e as interações entre funcionários e clientes (TAN; HAMID; CHEW, 2016; ZEITHAML; BITNER; GREMLER, 2014).

III. Medir as operações de serviço através de padrões definidos pelo cliente. Quando não existem padrões de serviço ou quando eles não refletem as expectativas dos clientes, a qualidade do serviço, tal como é percebida pelos clientes é impactada negativamente (KONI; ZAINAL; IBRAHIM, 2013; TAN; HAMID; CHEW, 2016; ZEITHAML; BITNER; GREMLER, 2014).

\subsubsection{Lacuna 3: a lacuna do desempenho do serviço}

A existência desta lacuna significa que há uma discrepância entre o projeto e os padrões de serviços orientados ao cliente e à entrega efetiva dos serviços. Mesmo quando as diretrizes existem para atender corretamente os clientes, o desempenho do serviço de alta qualidade não é uma certeza. As principais causas são as deficiências nas políticas de recursos humanos, fracasso ao equilibrar oferta e demanda, os clientes não desempenham seus papéis e os problemas com os intermediários nos serviços (TAN; HAMID; CHEW, 2016; GREEN, 2014; ZEITHAML; BITNER; GREMLER, 2014).

\subsubsection{Estratégias para o fechamento da Lacuna 3 - Desempenho do serviço:}

I. Alinhar as estratégias de recursos humanos da empresa em torno da excelência no serviço (TAN; HAMID; CHEW, 2016; ZEITHAML; BITNER; GREMLER, 2014).

II. Para oferecer efetivamente a qualidade do serviço, uma atenção considerável deve ser focada no recrutamento e contratação do pessoal adequado para o serviço. Uma vez que as pessoas são contratadas para fornecer um serviço de qualidade, elas precisam ser desenvolvidas através de treinamento contínuo para adquirir conhecimento técnico e habilidades interativas. As organizações também usam uma variedade de recompensas para manter os melhores funcionários, como salários mais altos, promoções e prêmios, que geralmente estão ligados ao desempenho do serviço (KONI; ZAINAL; IBRAHIM, 2013; TAN; HAMID; CHEW, 2016; ZEITHAML; BITNER; GREMLER, 2014). 


\section{REVISTA ENIAC PESQUISA}

III. Ajudar os clientes a entender como desempenhar seus papéis efetivamente. Às vezes, os clientes contribuem com a lacuna do desempenho do serviço porque não têm conhecimento de seus papéis e não sabem exatamente o que são em uma dada situação ou até mesmo porque não estão dispostos ou se sentem incapazes de realizar seu papel por algum motivo. Para reduzir esta lacuna, a organização precisa definir e comunicar claramente qual é o papel do cliente (a "descrição do trabalho" do cliente) e ajuda-lo a desempenhar esse papel (ZEITHAML; BITNER; GREMLER, 2014).

IV. Aplicar a integração efetiva e adequada da tecnologia para ajudar o desempenho do serviço. Para que os clientes sejam eficientes e eficazes no desempenho do serviço, muitas vezes é necessária uma tecnologia que facilite seus esforços (KONI; ZAINAL; IBRAHIM, 2013; TAN; HAMID; CHEW, 2016; ZEITHAML; BITNER; GREMLER, 2014).

\subsubsection{Lacuna 4: a lacuna da comunicação}

Pode haver uma falha em atender às expectativas dos clientes quando a comunicação sobre o serviço não coincide com o que é entregue. Esta lacuna concentra-se na diferença entre a prestação de serviços e o que é comunicado externamente aos clientes através de publicidade, preços e outras formas de comunicações tangíveis. As suas principais causas são a falta de comunicação integrada no marketing de serviços, a falta de comunicação ascendente, promessas excessivas, a comunicação horizontal inadequada e precificação inadequada (GREEN, 2014; TAN; HAMID; CHEW, 2016; ZEITHAML; BITNER; GREMLER, 2014).

\subsubsection{Estratégias para o fechamento da Lacuna 4 - Comunicação:}

I. Integrar comunicação de marketing de serviços. Isso garante que tudo e todos que enviam uma mensagem ou um sinal sobre o serviço, o fazem de forma consistente com o que os clientes esperam e com o que realmente é entregue. O desafio é que existe uma variedade de canais e modos de comunicação que enviam mensagens aos clientes, incluindo sites tradicionais, vendas pessoais, mala direta, mídia impressa, blogs, comunidades virtuais, publicidade em celular, etc. (TAN; HAMID; CHEW, 2016; ZEITHAML; BITNER; GREMLER, 2014).

II. Gerenciar as expectativas dos clientes de forma eficaz ao longo da experiência do serviço. Muitos serviços ocorrem durante um período de tempo prolongado 


\section{REVISTA ENIAC PESQUISA}

que pode significar horas, dias, semanas ou mesmo anos. Esses tipos de experiências de serviço estendido, muitas vezes mudam ao longo do tempo, variando a promessa de serviço original (tanto para o provedor quanto para o cliente) (TAN; HAMID; CHEW, 2016; ZEITHAML; BITNER; GREMLER, 2014).

Assim, é fundamental que a comunicação ao cliente também mude e evolua ao longo do tempo, para garantir que as expectativas e o desempenho do serviço correspondam (TAN; HAMID; CHEW, 2016; ZEITHAML; BITNER; GREMLER, 2014).

III. Desenvolver mecanismos de comunicação interna para que o cliente receba mensagens consistentes antes da venda e durante a entrega do serviço (TAN; HAMID; CHEW, 2016; ZEITHAML; BITNER; GREMLER, 2014).

As promessas excessivas podem ser perdidas rapidamente com a falha na entrega. A venda da marca dentro da empresa ajuda os funcionários a perceberem seu valor e a serem realistas sobre o que pode e deve ser prometido aos clientes (TAN; HAMID; CHEW, 2016; ZEITHAML; BITNER; GREMLER, 2014).

\subsection{GRAVIDADE DA FALHA}

A gravidade da falha do serviço refere-se à intensidade percebida por um cliente de um problema de serviço. Quanto mais intensa ou grave a falha do serviço, maior é a perda percebida pelo cliente (NIKBIN; HYUN, 2015; WEUN; BEATTY; JONES, 2004). A gravidade da falha é a extensão da perda experimentada por clientes a partir de uma falha a qual pode ser tangível, implicando perda de valor do serviço, representando a perda o tempo ou experiência social compartilhada (SMITH; BOLTON; WAGNER, 1999).

A gravidade de uma falha no serviço tem um efeito negativo na satisfação com a recuperação do serviço (MCQUILKEN, 2010; NIKBIN; HYUN, 2015; WEUN; BEATTY; JONES, 2004). As conclusões de Weun, Beatty e Jones (2004) demonstram que quanto mais grave for o problema do serviço, mais provável será a insatisfação do cliente.

Apesar da boa qualidade do desempenho do serviço passado, é provável que uma falha grave no serviço produza alguma perda percebida, resultando na diminuição na satisfação do cliente. Portanto, quanto mais intensa ou grave for a falha no serviço, maior será a perda percebida pelo cliente (NIKBIN; HYUN, 2015; WEUN; BEATTY; JONES, 2004). 


\section{REVISTA ENIAC PESQUISA}

As pesquisas de Krishna, Dangayach e Sharma (2014); Mccollough (2009) demonstram que quanto maior for a gravidade da falha do serviço, será necessário um maior desempenho de recuperação para converter a insatisfação dos clientes em satisfação e quanto mais difícil deve ser para o provedor de serviços obter recuperação total. Da mesma forma, Migacz e Petrick (2017); Fayos-Gardó et al. (2017) reconhecem uma relação significativa entre a gravidade da falha do serviço e as percepções de recuperação do serviço.

\section{METODOLOGIA}

Esta é uma pesquisa descritiva, com abordagem qualitativa e quantitativa e a pesquisa foi realizada por meio de um survey. O público-alvo são clientes de bancos que vivenciaram algum problema com os serviços prestados nos últimos 12 meses e que tenham comunicado esse problema ao banco e esse tenha tentado resolver esse problema na prestação de serviços.

No início do questionário, foram inseridas três perguntas de crivo: (1) O banco do qual o senhor(a) é cliente apresentou alguma falha importante na prestação de serviços nos últimos 12 meses? (2) O senhor(a) comunicou ou reclamou dessa falha com o banco? (3) O banco tentou de alguma forma resolver esse problema ou essa falha na prestação de serviços? Caso alguma das perguntas de crivo fosse respondida com "NÃO", o questionário era encerrado e descartado da análise. Após as três primeiras questões, o respondente relatou de forma sucinta - qual foi o tipo de problema de serviço que ele teve.

$\mathrm{O}$ respondente avaliou a gravidade da falha no serviço atribuindo de 0 "falha pequena" até 10 "falha grave". A coleta ocorreu por meio de questionários on-line, através da plataforma Google Forms e também questionários impressos. A amostra da pesquisa é uma amostra de conveniência. Os respondentes foram contatos por meio de Facebook, Instagram, Linkeind, Whatsapp e e-mail. Foram coletados 1199 questionários no total, sendo 897 deles online e 302 deles impressos. Em função das perguntas de crivo foram descartados 549 questionários online e 59 questionários impressos. Foi necessário retirar 12 questionários impressos por dados faltantes. Assim, foram considerados como válidos 579 questionários, sendo 348 deles online e 231 deles impressos.

A análise dos dados foi realizada por meio de análise de conteúdo (BARDIN, 2009).

Revista ENIAC Pesquisa, Guarulhos (SP), V.8, n.2, jul.- dez. 2019. 


\section{REVISTA ENIAC PESQUISA}

\section{ANÁLISE DE DADOS}

Os resultados relacionados à pesquisa são apresentados na Tabela 1, a qual exibe os principais problemas relatados pelos clientes de bancos.

Tabela 1: Tipos de problema relatado pelo cliente

\begin{tabular}{|c|c|c|c|c|}
\hline \multicolumn{2}{|c|}{ Tipo de problema relatado pelo cliente } & \multirow{2}{*}{$\begin{array}{c}\begin{array}{c}\text { Frequência } \\
\text { absoluta }\end{array} \\
149\end{array}$} & \multirow{2}{*}{$\begin{array}{c}\begin{array}{c}\text { Frequência } \\
\text { relativa }\end{array} \\
25,7 \%\end{array}$} & \multirow{2}{*}{$\begin{array}{c}\begin{array}{c}\text { Média da } \\
\text { gravidade da } \\
\text { falha }\end{array} \\
8,09\end{array}$} \\
\hline 1 & Cobrança indevida de taxa/tarifa bancária & & & \\
\hline 2 & Compra/transação não reconhecida & 56 & $9,7 \%$ & 8,30 \\
\hline 3 & Pagamento agendado, mas não realizado pelo banco & 44 & $7,6 \%$ & 8,32 \\
\hline 4 & Duplicidade de pagamento & 33 & $5,7 \%$ & 8,42 \\
\hline 5 & Juros abusivos & 28 & $4,9 \%$ & 8,57 \\
\hline 6 & APP/Internet banking falhando & 25 & $4,3 \%$ & 7,92 \\
\hline 7 & Bloqueio indevido do cartão & 22 & $3,8 \%$ & 8,23 \\
\hline 8 & Dificuldade para conseguir atendimento & 22 & $3,8 \%$ & 7,82 \\
\hline 9 & Atraso no envio de cartão & 19 & $3,3 \%$ & 7,58 \\
\hline 10 & Mau atendimento & 18 & $3,1 \%$ & 7,78 \\
\hline 11 & Protesto indevido (SPC indevidamente /Serasa) & 15 & $2,6 \%$ & 8,80 \\
\hline 12 & Cartão clonado & 13 & $2,2 \%$ & 8,08 \\
\hline 13 & Saque não realizado, mas debitado na conta & 13 & $2,2 \%$ & 8,23 \\
\hline 14 & Não envio de fatura/boleto & 11 & $1,9 \%$ & 8,09 \\
\hline 15 & Venda casada & 9 & $1,6 \%$ & 7,56 \\
\hline 16 & Bloqueio indevido da conta & 6 & $1,1 \%$ & 9,17 \\
\hline 17 & Depósito realizado, mas o valor não consta na conta & 6 & $1,1 \%$ & 9,50 \\
\hline 18 & Insatisfação com o limite do cartão & 6 & $1,1 \%$ & 8,33 \\
\hline 19 & Dificuldade para conseguir cancelar serviço & 6 & $1,1 \%$ & 8,17 \\
\hline 20 & $\begin{array}{l}\text { Dificuldade para realizar a portabilidade para outro } \\
\text { banco }\end{array}$ & 5 & $0,9 \%$ & 8,80 \\
\hline 21 & Dificuldade para resgate de aplicação & 5 & $0,9 \%$ & 9,20 \\
\hline 22 & Aplicação de valor sem autorização do cliente & 5 & $0,9 \%$ & 9,20 \\
\hline 23 & Banco liquidado pelo Banco Central & 4 & $0,7 \%$ & 9,25 \\
\hline 24 & Bloqueio indevido da margem de consignado & 4 & $0,7 \%$ & 9,25 \\
\hline 25 & Cheque sustado, mas compensado & 4 & $0,7 \%$ & 8,75 \\
\hline 26 & Contratação de serviços sem solicitação & 3 & $0,5 \%$ & 10,00 \\
\hline 27 & Contrato elaborado com erros & 3 & $0,5 \%$ & 9,33 \\
\hline 28 & Dificuldade para conseguir cancelar cartão & 3 & $0,5 \%$ & 6,67 \\
\hline 29 & Dificuldade para realizar aplicação & 3 & $0,5 \%$ & 9,67 \\
\hline 30 & Dificuldade sacar o FGTS & 3 & $0,5 \%$ & 5,67 \\
\hline
\end{tabular}


31 Pagamento da alienação, mas sem baixa no gravame

\section{3}

32 Porta giratória com mau funcionamento

33 Propaganda enganosa

34 Ativação de conta corrente sem autorização do cliente

35 Atraso na liberação do financiamento

36 Atraso na TED (transferência eletrônica disponível)

37 Caixas eletrônicos sem funcionamento

38 Cheque do cliente devolvido indevidamente

39 Concessão de crédito consignado sem autorização

40 Dados fraudados

41 Demora na entrega de cheque devolvido

42 Descumprimento de prazo

43 Dificuldade para liquidar empréstimo

44 Dificuldade para recuperar a senha

45 Falha no cadastramento de biometria

46 Faltam informações sobre as regras do cartão de crédito

47 Fatura paga, mas não liberou o limite do cartão

48 Pagamento de financiamento apenas presencial

49 Suspendeu a emissão de talão de cheques

50

TED (transferência electronica disponível) não realizada

TOTAL

\section{3}

3

2

2

2

2

2

2

2

2

2

2

2

2

2

2

1

1

2

$0,5 \%$

$0,5 \%$

9,00

$0,5 \%$

7,67

2

$$
0,3 \%
$$

7,33

$0,3 \%$

$0,3 \%$

9,00

8,00

$0,3 \%$

8,00

$0,3 \%$

8,50

2

$$
0,3 \%
$$

10,00

$0,3 \%$

9,00

$0,3 \% \quad 9,50$

$0,3 \% \quad 6,00$

$0,3 \% \quad 8,50$

$0,3 \% \quad 8,50$

$0,2 \% \quad 6,00$

$0,2 \% \quad 4,00$

$0,2 \% \quad 8,00$

$0,2 \% \quad 7,00$

$0,2 \% \quad 6,00$

$0,2 \% \quad 7,00$

$1 \quad 0,2 \% \quad 10,00$

Fonte: Dados da pesquisa (2018).

Conforme pode ser observado na Tabela 1, os problemas relatados pelos clientes foram classificados em 50 tipos, nos quais aproximadamente metade (50\%) dos problemas se concentraram nos 5 primeiros tipos: (I) Cobrança indevida de taxas/tarifas bancárias; (II) Compra/transação não reconhecida; (III) Pagamento agendado, mas não realizado pelo banco; (IV) Duplicidade de pagamento; e (V) Juros abusivos. Também vale destacar que $80 \%$ dos problemas relatados pelos clientes se concentraram nos 15 primeiros itens.

Em relação à categoria dos problemas, ela pode ser dividida como: (1) Problemas com descontos indevidos; (2) Problemas com erros de processamento; (3) Problemas com atendimento; (4) Problemas com falhas de segurança; e (5) Problemas diversos.

Revista ENIAC Pesquisa, Guarulhos (SP), V.8, n.2, jul.- dez. 2019. 


\section{REVISTA ENIAC PESQUISA}

Tabela 2: Tipos de "Problemas descontos indevidos" relatados pelos clientes.

\begin{tabular}{lccc}
\hline $\begin{array}{l}\text { Categoria "Problemas com descontos indevidos" } \\
\text { Tipo de problema relatado pelo cliente }\end{array}$ & $\begin{array}{c}\text { Frequência } \\
\text { absoluta }\end{array}$ & $\begin{array}{c}\text { Frequência } \\
\text { relativa }\end{array}$ & $\begin{array}{c}\text { Média da } \\
\text { gravidade da falha }\end{array}$ \\
\hline Cobrança indevida de taxa/tarifa bancária & 149 & $25,7 \%$ & 8,09 \\
Duplicidade de pagamento & 33 & $5,7 \%$ & 8,42 \\
Juros abusivos & 28 & $4,9 \%$ & 8,57 \\
Saque não realizado, mas debitado na conta & 13 & $2,2 \%$ & 8,23 \\
Depósito realizado, mas o valor não consta na conta & 6 & $1,1 \%$ & 9,50 \\
Aplicação de valor sem autorização do cliente & 5 & $0,9 \%$ & 9,20 \\
Cheque sustado, mas compensado & 4 & $0,7 \%$ & 8,75 \\
Contratação de serviços sem solicitação & 3 & $0,5 \%$ & 10,00 \\
Concessão de crédito consignado sem autorização & 2 & $0,3 \%$ & 9,00 \\
\hline \multicolumn{1}{c}{ TOTAL } & $\mathbf{2 4 3}$ & $\mathbf{4 2 , 0 \%}$ & $\mathbf{8 , 8 6}$
\end{tabular}

Fonte: Dados da pesquisa (2018).

A categoria do tipo de reclamação que apresentou o maior valor no geral foi a categoria "Problemas com descontos indevidos". Verifica-se que essa categoria apresenta $42 \%$ do total de reclamações dos clientes de bancos. Nessa categoria, o item principal foi a "cobrança indevida de taxas/tarifas bancárias" com mais da metade das ocorrências nessa categoria. Também aparecem com algumas citações de "duplicidade de pagamento" e a cobrança de "juros abusivos".

A próxima categoria encontrada na análise de conteúdo é a categoria "Problemas com erros de processamento" (Tabela 3).

Revista ENIAC Pesquisa, Guarulhos (SP), V.8, n.2, jul.- dez. 2019. 


\section{REVISTA ENIAC PESQUISA}

Tabela 3: Tipos de "Problemas com erros de processamento" relatados pelos clientes

\begin{tabular}{lccc}
\hline $\begin{array}{l}\text { Categoria "Problemas com erros de processamento" } \\
\text { Tipo de problema relatado pelo cliente }\end{array}$ & $\begin{array}{c}\text { Frequência } \\
\text { absoluta }\end{array}$ & $\begin{array}{c}\text { Frequên } \\
\text { cia } \\
\text { relativa }\end{array}$ & $\begin{array}{c}\text { Média da } \\
\text { gravidade da } \\
\text { falha }\end{array}$ \\
\hline Pagamento agendado, mas não realizado pelo banco & 44 & $7,6 \%$ & 8,32 \\
APP/Internet banking falhando & 25 & $4,3 \%$ & 7,92 \\
Bloqueio indevido do cartão & 22 & $3,8 \%$ & 8,23 \\
Atraso no envio de cartão & 19 & $3,3 \%$ & 7,58 \\
Protesto indevido (SPC/Serasa) & 15 & $2,6 \%$ & 8,80 \\
Não envio da fatura/boleto & 11 & $1,9 \%$ & 8,09 \\
Bloqueio indevido da conta & 6 & $1,1 \%$ & 9,17 \\
Bloqueio indevido da margem de consignado & 4 & $0,7 \%$ & 9,25 \\
Pagamento da alienação, mas sem baixa no gravame & 3 & $0,5 \%$ & 9,00 \\
Ativação de conta corrente sem autorização do cliente & 2 & $0,3 \%$ & 9,00 \\
Atraso na TED (transferência eletrônica disponível) & 2 & $0,3 \%$ & 8,00 \\
Cheque do cliente devolvido indevidamente & 2 & $0,3 \%$ & 10,00 \\
Fatura paga, mas não liberado o limite do cartão & 1 & $0,2 \%$ & 7,00 \\
Suspendeu a emissão de talão de cheques & 1 & $0,2 \%$ & 7,00 \\
TED (transferência eletrônica disponível) não realizada & 1 & $0,2 \%$ & 10,00 \\
\hline TOTAL & $\mathbf{1 5 8}$ & $\mathbf{2 7 , 3 \%}$ & $\mathbf{8 , 4 9}$ \\
\hline
\end{tabular}

Fonte: Dados da pesquisa (2018).

A categoria do tipo de reclamação, que apresentou o segundo maior valor no geral, foi a categoria de "Problemas com erros de processamento" que apresenta 27,3\% do total de reclamações dos clientes de bancos. Nessa categoria, o item principal foi o "pagamento agendado, mas não realizado pelo banco" com $7,6 \%$ do total de todos os tipos de reclamações.

A próxima categoria encontrada na análise de conteúdo é a categoria "Problemas com atendimento" (Tabela 4).

Revista ENIAC Pesquisa, Guarulhos (SP), V.8, n.2, jul.- dez. 2019. 


\section{REVISTA ENIAC PESQUISA}

Tabela 4: Tipos de "Problemas com atendimento" relatados pelos clientes.

\begin{tabular}{lccc}
\hline $\begin{array}{l}\text { Categoria "Problemas com atendimento" } \\
\text { Tipo de problema relatado pelo cliente }\end{array}$ & $\begin{array}{c}\text { Frequência } \\
\text { absoluta }\end{array}$ & $\begin{array}{c}\text { Frequência } \\
\text { relativa }\end{array}$ & $\begin{array}{c}\text { Média da } \\
\text { gravidade } \\
\text { da falha }\end{array}$ \\
\hline Dificuldade para conseguir atendimento & 22 & $3,8 \%$ & 7,82 \\
Mau atendimento & 18 & $3,1 \%$ & 7,78 \\
Dificuldade para conseguir cancelar serviço & 6 & $1,1 \%$ & 8,17 \\
Dificuldade para realizar a portabilidade para outro banco & 5 & $0,9 \%$ & 8,80 \\
Dificuldade para resgatar aplicação & 5 & $0,9 \%$ & 9,20 \\
Contrato elaborado com erros & 3 & $0,5 \%$ & 9,33 \\
Dificuldade para conseguir cancelar cartão & 3 & $0,5 \%$ & 6,67 \\
Dificuldade para realizar aplicação & 3 & $0,5 \%$ & 9,67 \\
Dificuldade sacar o FGTS & 3 & $0,5 \%$ & 5,67 \\
Atraso na liberação do financiamento & 2 & $0,3 \%$ & 8,00 \\
Caixas eletrônicos sem funcionamento & 2 & $0,3 \%$ & 8,50 \\
Demora na entrega de cheque devolvido & 2 & $0,3 \%$ & 6,00 \\
Descumprimento de prazo & 2 & $0,3 \%$ & 8,50 \\
Dificuldade para liquidar empréstimo & 2 & $0,3 \%$ & 8,50 \\
Dificuldade para recuperar a senha & 1 & $0,2 \%$ & 6,00 \\
Falha no cadastramento de biometria & 1 & $0,2 \%$ & 4,00 \\
Falta informações sobre as regras do cartão de crédito & 1 & $0,2 \%$ & 8,00 \\
Pagamento de financiamento apenas presencial & 1 & $0,2 \%$ & 6,00 \\
\hline \multicolumn{1}{c}{ TOTAL } & $\mathbf{8 2}$ & $\mathbf{1 4 , 1 \%}$ & $\mathbf{7 , 5 9}$ \\
\hline Fonte: Dados da pesquis & & & \\
\hline
\end{tabular}

Fonte: Dados da pesquisa (2018).

A categoria do tipo de reclamação que apresentou o terceiro maior valor no geral foi a categoria de "Problemas com atendimento" que apresenta 14,1\% do total de reclamações dos clientes de bancos. Nessa categoria, o item principal foi a "dificuldade para conseguir atendimento" com 3,8\% do total de todos os tipos de reclamações.

A próxima categoria encontrada na análise de conteúdo é a categoria "Problemas com falhas de segurança" (Tabela 5).

Revista ENIAC Pesquisa, Guarulhos (SP), V.8, n.2, jul.- dez. 2019. 


\section{REVISTA ENIAC PESQUISA}

Tabela 5: Tipos de "Problemas com falhas de segurança" relatados pelos clientes

\begin{tabular}{lccc}
\hline $\begin{array}{l}\text { Categoria "Problemas com falhas de segurança" } \\
\text { Tipo de problema relatado pelo cliente }\end{array}$ & $\begin{array}{c}\text { Frequência } \\
\text { absoluta }\end{array}$ & $\begin{array}{c}\text { Frequência } \\
\text { relativa }\end{array}$ & $\begin{array}{c}\text { Média da } \\
\text { gravidade da falha }\end{array}$ \\
\hline Compra/transação não reconhecida & 56 & $9,7 \%$ & 8,30 \\
Cartão clonado & 13 & $2,2 \%$ & 8,08 \\
Banco liquidado pelo Banco Central & 4 & $0,7 \%$ & 9,25 \\
Dados fraudados & 2 & $0,3 \%$ & 9,50 \\
\hline TOTAL & $\mathbf{7 5}$ & $\mathbf{1 2 , 9 \%}$ & $\mathbf{8 , 7 8}$ \\
\hline
\end{tabular}

Fonte: Dados da pesquisa (2018).

A categoria do tipo de reclamação que apresentou o quarto maior valor no geral, foi a categoria de "Problemas com falhas de segurança" que apresenta 12,9\% do total de reclamações dos clientes de bancos. Nessa categoria, o item principal foi a "compra/transação não reconhecida" com $9,7 \%$ do total de todos os tipos de reclamações.

A próxima categoria encontrada na análise de conteúdo é a "Problemas diversos".

Tabela 6: Tipos de "Problemas diversos" relatados pelos clientes

\begin{tabular}{lccc}
\hline $\begin{array}{l}\text { Categoria "Problemas diversos" } \\
\text { Tipo de problema relatado pelo cliente }\end{array}$ & $\begin{array}{c}\text { Frequência } \\
\text { absoluta }\end{array}$ & $\begin{array}{c}\text { Frequência } \\
\text { relativa }\end{array}$ & $\begin{array}{c}\text { Média da gravidade da } \\
\text { falha }\end{array}$ \\
\hline Venda casada & 9 & $1,6 \%$ & 7,56 \\
Insatisfação com o limite do cartão & 6 & $1,1 \%$ & 8,33 \\
Porta giratória com mau funcionamento & 3 & $0,5 \%$ & 7,67 \\
Propaganda enganosa & 3 & $0,5 \%$ & 7,33 \\
\hline TOTAL & $\mathbf{2 1}$ & $\mathbf{3 , 7}$ & $\mathbf{7 , 7 2}$ \\
\hline
\end{tabular}

Fonte: Dados da pesquisa (2018).

A categoria do tipo "Problemas diversos" (Tabela 6) apresenta 3,7\% do total de reclamações dos clientes de bancos, sendo o principal item a "venda casada".

\section{CONCLUSÃO}

Por meio deste trabalho foi possível verificar as ações propostas de prevenção de falhas em serviços bancários com base nas lacunas de falha na qualidade do serviço e nas estratégias para o fechamento das lacunas.

Além disso, foi possível classificar os principais problemas relatados pelos clientes de bancos que vivenciaram experiências negativas com serviços bancários, identificando a

Revista ENIAC Pesquisa, Guarulhos (SP), V.8, n.2, jul.- dez. 2019. 


\section{REVISTA ENIAC PESQUISA}

gravidade da falha percebida pelos clientes de bancos para os principais problemas relatados, correlacionando os problemas relatados pelos clientes com as lacunas de falha na qualidade dos serviços bancários e descrevendo estratégias para o fechamento das lacunas de falha na qualidade dos serviços bancários.

Portanto, como implicações gerências desta pesquisa, sugere-se aos gestores de bancos, ouvir os clientes de múltiplas formas, através da pesquisa com o cliente e da comunicação ascendente dos funcionários, criar relacionamentos através da compreensão e atendimento às necessidades dos clientes, saber e agir sobre o que os clientes esperam quando experimentam uma falha no serviço.

Sugere-se também, empregar práticas bem definidas de desenvolvimento de novos serviços e inovação para a concepção de serviços, compreender a experiência do cliente e desenhar todos os elementos dessa experiência, de forma a atender ou exceder as expectativas do cliente.

Além disso, garantir que os funcionários estejam dispostos e capazes de oferecer serviços de qualidade e que eles sejam motivados a atuar para o cliente de forma orientada, definir e comunicar claramente qual é o papel do cliente no processo, aplicar a integração efetiva e adequada da tecnologia para ajudar no aumento do desempenho do serviço.

Recomenda-se aos gestores de bancos, integrar a comunicação de marketing de serviços, para que tudo e todos que enviam uma mensagem sobre o serviço o façam de forma consistente com o que os clientes esperam e com o que realmente é entregue, gerenciar as expectativas dos clientes de forma eficaz ao longo da experiência do serviço, desenvolver mecanismos de comunicação para que o cliente receba mensagens consistentes antes da venda e durante a entrega do serviço.

A principal limitação dessa pesquisa é a amostragem por conveniência usada, a qual faz com que os resultados encontrados não reflitam a população de uma forma generalizada.

\section{REFERÊNCIAS}

BARDIN, L. Análise de conteúdo. Lisboa: Edições 70, 2009.

BATESON, J. E. G.; HOFFMAN, K. D. Princípios de marketing de serviços: conceitos, estratégias e casos. São Paulo: Cengage Learning, 2016. 
CHEN, P.-T.; HU, H.-H. S. The mediating role of relational benefit between service quality and customer loyalty in airline industry. Total Quality Management \& Business Excellence, v. 24, n. 9-10, p. 1084-1095, 2013.

FAYOS-GARDÓ, T. et al. Analysing the fulfilment of service recovery paradox in retailing. The International Review of Retail, Distribution and Consumer Research, v. 27, n. 4, p. 1-21, 2017.

GREEN, P. Measuring service quality in higher education: A South African case study. Journal of International Education Research, v. 10, n. 2, p. 131, 2014.

HESS JR, R. L. The impact of firm reputation and failure severity on customers' responses to service failures. Journal of Services Marketing, v. 22, n. 5, p. 385-398, 2008.

KONI, A.; ZAINAL, K.; IBRAHIM, M. An assessment of the services quality of Palestine higher education. International Education Studies, v. 6, n. 2, p. 33-48, 2012.

KRISHNA, A.; DANGAYACH, G. S.; SHARMA, S. Service recovery paradox: the success parameters. Global Business Review, v. 15, n. 2, p. 263-277, 2014.

KURTULMUŞOĞLU, F. B.; PAKDIL, F. Combined analysis of service expectations and perceptions in lodging industry through quality function deployment. Total Quality Management \& Business Excellence, v. 28, n. 11-12, p. 1393-1413, 2016.

LIMA, K. R.; MOURA, L. R. C; SOUKI, G. Q. Proposição e teste de escala de avaliação da qualidade do metrô. Revista Pretexto, v. 16, n. 3, p. 21-40, 2015.

MIGACZ, S. J.; ZOU, S.; PETRICK, J. F. The "terminal" effects of service failure on airlines: examining service recovery with justice theory. Journal of Travel Research, $v$. 57, n. 1, p. 83-98, 2018.

MCCOLLOUGH, M. A. The recovery paradox: The effect of recovery performance and service failure severity on post-recovery customer satisfaction. Academy of Marketing Studies Journal, v. 13, n. 1, p. 89-104, 2009.

MCQUILKEN, L. The influence of failure severity and employee effort on service recovery in a service guarantee context. Australasian Marketing Journal, v.18, n.4, p.214-221, 2010.

MOURA, L. R. C. et al. Avaliação da qualidade de serviços utilizando a escala Servqual: o estudo de caso Matermed. Revista Reuna, v. 12, n. 3, p. 21-36, 2010.

MOURA, L. R. C. et al. Test and validity of the Brand Resonance Model's. Revista Gestão \& Tecnologia, v. 19, n. 1, p. 4-24, 2019. 
NIKBIN, D.; HYUN, S. S. An empirical study of the role of failure severity in service recovery evaluation in the context of the airline industry. Review of Managerial Science, v. 9 , n. 4 , p. 731-749, 2015.

PARASURAMAN, A.; ZEITHAML, V.; BERRY, L. A conceptual model of service quality and its implications for future research. Journal of Marketing, v. 49, n. 3, p. 41-50, 1985.

SMITH, A. K.; BOLTON, R. N.; WAGNER, J. A model of customer satisfaction with service encounters involving failure and recovery. Journal of Marketing Research, v. 36, p. 356-372, 1999.

REIMANN, M.; LÜNEMANN, U. F.; CHASE, R. B. Uncertainty avoidance as a moderator of the relationship between perceived service quality and customer satisfaction. Journal of Service Research, v. 11, n. 1, p. 63-73, 2008.

TAN, L-H; HAMID, S-R; CHEW,B-C. Service quality audit based on conceptual gaps model of service quality: a case study of top three largest local bank in Malaysia. International Journal of Productivity and Quality Management, v. 18, n. 1, p. 99-115, 2016.

WEUN, S.; BEATTY, S. E.; JONES, M. A. The impact of service failure severity on service recovery evaluations andpost-recovery relationships. Journal of Services Marketing, v. 18, n. 2, p. 133-146, 2004.

ZEITHAML, V. A.; BERRY, L. L.; PARASURAMAN, A. The nature and determinants of customer expectations of service. Journal of the Academy of Marketing Science, v. 21, n. 1, p. 1-12, 1993.

ZEITHAML, V. A.; BITNER, M. J.; GREMLER, D. D. Marketing de Serviços. Porto Alegre: Artmed, 2014. 